\title{
Quantum communication with correlated nonclassical states
}

\author{
S. F. Pereira, ${ }^{*}$ Z. Y. Ou, ${ }^{\dagger}$ and H. J. Kimble \\ Norman Bridge Laboratory of Physics 12-33, California Institute of Technology, Pasadena, California 91125 \\ (Received 19 April 1993; revised manuscript received 23 March 2000; published 15 September 2000)
}

\begin{abstract}
Nonclassical correlations between the quadrature-phase amplitudes of two spatially separated optical beams are exploited to realize a two-channel quantum communication experiment with a high degree of immunity to interception. For this scheme, either channel alone can have an arbitrarily small signal-to-noise ratio (SNR) for transmission of a coherent "message." However, when the transmitted beams are combined properly upon authorized detection, the encoded message can in principle be recovered with the original SNR of the source. An experimental demonstration has achieved a $3.2 \mathrm{~dB}$ improvement in SNR over that possible with correlated classical sources. Extensions of the protocol to improve its security against eavesdropping are discussed.
\end{abstract}

PACS number(s): 03.67.Dd, 42.50.-p

\section{INTRODUCTION}

Principal motivations for the investigation of manifestly quantum or nonclassical states of the electromagnetic field have been their possible exploitation for optical communication [1-3] and for enhanced measurement sensitivity [4]. For example, relative to a coherent state, the reduced quantum fluctuations associated with squeezed and number states offer potential for improving channel capacity in the transmission of information [3]. Squeezed states of light have been widely employed to achieve measurement sensitivity beyond the standard quantum limits in applications such as precision interferometry [5] the detection of directly encoded amplitude modulation [6], atomic spectroscopy [7], and quantum noise reduction in optical amplification [8]. Likewise, nonclassical correlations for the amplitudes of spatially separated beams have been exploited in diverse situations, including demonstrations of the Einstein-Podolsky-Rosen (EPR) paradox for continuous variables [9], of quantum nondemolition detection $[10,11]$, and of a quantum-optical tap [12].

Within the broader setting of quantum information science, there has been growing interest and important progress concerning the prospects for quantum information processing with continuous quantum variables, including universal quantum computation [13], quantum error correction [1416], and entanglement purification $[17,18]$. Theories for quantum teleportation of continuous quantum variables in an infinite dimensional Hilbert space have been developed [1922], including for broad bandwidth teleportation [23], and for teleportation of atomic wave packets [24]. This formalism has also been applied to super-dense quantum coding [25]. On an experimental front, these developments in QIS led to the first bona fide demonstration of quantum teleportation, which was carried out by exploiting nonclassical

\footnotetext{
*Present address: Faculty of Applied Sciences, Optics Research Group, Delft University of Technology, Lorentzweg 1, 2628 CJ Delft, The Netherlands.

${ }^{\dagger}$ Present address: Department of Physics, Indiana UniversityPurdue University at Indianapolis, 402 N Blackford Street, Indianapolis, IN 46202.
}

states of light in conjunction with continuous quantum variables [26,27].

Against this backdrop the focus of attention in this paper is optical communication in two channels with quantum correlated light fields and the associated quadrature amplitudes [28]. The goal is to explore the extension of quantum cryptography from the usual setting of discrete variables as pioneered by Bennett and colleagues [29] (e.g., photon polarization as in the experiments of Refs. [30-33]) into the realm of continuous quantum variables (e.g., the complex amplitude of the electromagnetic field). Apart from our paper, several related schemes for quantum cryptography based upon continuous variables have recently been analyzed, including a single-beam scheme with squeezed light [34] as well dualbeam schemes with shared entanglement $[35,36]$. However, we stress at the outset that neither for our scheme nor for any of these other protocols, can any claim about absolute security be made. Rather, we suggest that these protocols (and suitable extensions thereof) are worthy candidates for more detailed analyses. Such an undertaking would involve various important matters of principle as well as practice for continuous quantum variables, and might hopefully lead to security proofs such as have recently emerged in the case of discrete variables [37-39].

As illustrated in Fig. 1, the basic idea in our scheme is to construct a "transmitter,' which combines a coherent signal of amplitude $\epsilon / t$ (the "message") with the large fluctuating fields generated in nondegenerate optical parametric amplification (the "noise'") [28]. The message and the noise are superimposed at mirror $M$ with transmission coefficient $t$ $\ll 1$. Note that although each of the two transmitted beams along channels $(A, B)$ has large phase insensitive fluctuations that are individually indistinguishable from a thermal source [40], the quadrature-phase amplitudes of the two beams can be quantum copies of one another $[9,41]$, and in fact form an entangled EPR state $[42,43]$. Hence proper subtraction of the photocurrents at the "receiver' can result in the faithful reconstruction of the encoded message even though the signalto-noise ratios $R_{j}(j=A, B)$ during transmission are individually much less than one. Indeed, in a lossless system with large parametric gain, the signal-to-noise ratio of the reconstructed message $R_{t}$ can approach the signal-to-noise ratio $R_{0}$ of the original message $\left(\epsilon^{2} / t^{2}\right)$, which was written 


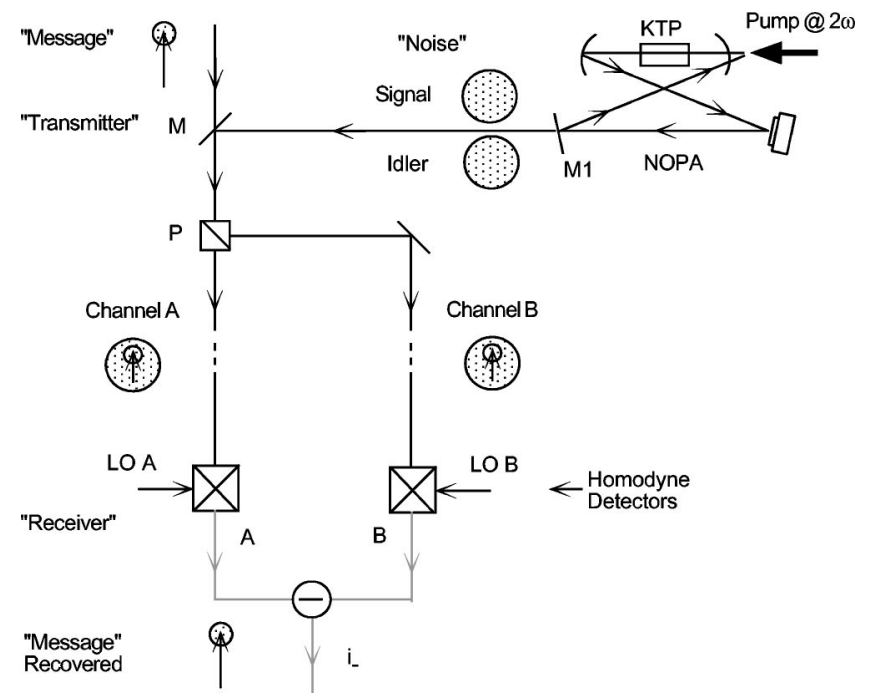

FIG. 1. Principal components of the experiment showing the "transmitter," where a message $\epsilon$ is combined with noise fields from a nondegenerate optical parametric amplifier (NOPA) at mirror $M$. The orthogonally polarized signal and idler beams are separated by polarizer $P$, and then propagate along independent channels $(A, B)$ to two separate balanced homodyne detectors that form the "receiver.' In the figure, arrows represent coherent amplitudes of various fields, while the shaded circles are meant to indicate their fluctuations.

in the transmitter as a coherent state before the mirror $M$ in Fig. 1. Note that the individual channels $(A, B)$ have a high degree of immunity to unauthorized interception since the signal-to-noise ratios $R_{A, B}$ in these channels are each very small. Furthermore, any attempt to extract information from the $(A, B)$ channels will reveal itself either by a decrease in $R_{t}$ (classical extraction) or by an increase in the fluctuations of the orthogonal quadrature amplitude (quantum extraction).

In addition to achieving a faithful reconstruction of the message transmitted through $M$ to the receiver, note that the scheme of Fig. 1 also preserves to a high degree the signalto-noise ratio for the original message beam that reflects from $M$. More specifically, for high gain and for losses dominated by the transmission coefficient of $M$, the signal-tonoise ratio $R_{r}$ for the reflected beam can approach $R_{0}$ for the original message. In this limit, we then have that the information transfer coefficient $\mathrm{T} \equiv\left(R_{r}+R_{t}\right) / R_{0} \rightarrow 2$, where 0 $\leqslant T \leqslant 1$ for classical devices and $1<T \leqslant 2$ for manifestly quantum or nonclassical situations [12]. Hence the scheme depicted in Fig. 1 acts as a quantum optical tap in the fashion originally discussed by Shapiro [44]. It provides a received (or "tapped") message with a signal-to-noise ratio equal to that of the input $\left(R_{t} / R_{0}\right) \rightarrow 1$, while simultaneously transmitting an output field with signal-to-noise ratio equal to that of the input $\left(R_{r} / R_{0}\right) \rightarrow 1$.

Of course similar schemes for two-channel communication can be implemented with correlated classical noise sources (i.e., thermal light), each with large fluctuations that "hide" the message $\epsilon$ during transmission. However, with classical sources of whatever type, only excess fluctuations can be subtracted; the quantum fluctuations at the vacuum- state level will remain unchanged and will enforce a noise floor for information transmission and extraction. For the case illustrated in Fig. 1, this noise floor for the message at the receiver is given by the sum of independent vacuum fluctuations from fields in channels $(A, B)$ and sets a fundamental noise level of " 2 ", (with " 1 "' as the individual vacuum-state limits for the two channels). Here we adopt the usual convention for the demarcation between classical and nonclassical correlations in terms of the behavior the Glauber-Sudarshan phase-space function [41]. Hence for the case illustrated in Fig. 1 but with classical input fields, the signal-to-noise ratio $R_{t}^{\prime}$ for the detected message at the receiver is given by $R_{t}^{\prime} \simeq|\epsilon|^{2} \ll R_{t}$. In fact, for classical inputs, we have that $T^{\prime} \equiv R_{t}^{\prime}+R_{r}^{\prime} \leqslant 1$, and the system no longer functions as a quantum optical tap. Furthermore, the individual channels $(A, B)$ are not protected from unauthorized eavesdropping, since information can be extracted from these channels with impunity for classical noise much greater than the vacuum-state limit.

Apart from these considerations related to secure communication and quantum optical tapping, the configuration of Fig. 1 can also be viewed as a means to realize super-dense quantum coding [45] for continuous quantum variables [25]. Here, the message $\epsilon / t$ is again encoded at the mirror $M$, but now in a single channel corresponding to one component of the entangled EPR state (e.g., channel $A$ ). This combination of the message and the fluctuations from one component of the nondegenerate optical parametric amplifier (NOPA) are transmitted to the receiving station where they are combined with the second component of the entangled output of the NOPA that has been independently transmitted (e.g., along channel $B$ ). The signal is then decoded by combining the outputs of the two channels in a fashion similar to that shown in Fig. 1 as discussed in more detail in Ref. [25]. The principal distinctions between this dense coding scheme and the aforementioned dual channel arrangement are (1) the message is encoded in a single component of the entangled EPR beam instead of symmetrically in both and (2) the received beams from paths $(A, B)$ must be physically recombined, with the phases of the local oscillators $(A, B)$ at the receiving station offset by $\pi / 2$. Recall that for dense coding in its canonical form [45], no signal modulation is applied to the second (i.e., channel $B$ ) component of the entangled state, so that it carries no information by itself.

In subsequent sections of this paper, we describe in more detail the implementation of this general discussion about quantum communication with correlated nonclassical fields. In our experiment, we have been able to demonstrate an improvement in signal-to-noise ratio by a factor of 2.1 over that possible with any classical source (that is, $\left.10 \log \left[R_{t} / R_{t}^{\prime}\right]=3.2 \mathrm{~dB}\right)$ and have succeeded in suppressing the noise of the difference photocurrent $i_{-} \equiv i_{A}-i_{B}$ below that associated with the vacuum fluctuations of even a single beam, thus making possible transmission with $|\epsilon|^{2}<1$. Quantum dense coding would thereby be enabled with the aforementioned changes in the overall experimental protocol. We conclude with a discussion of possible extensions for enhanced security against unauthorized eavesdropping. 


\section{IMPLEMENTATION BY NONDEGENERATE PARAMETRIC AMPLIFICATION}

As illustrated in Fig. 1, correlated nonclassical states for our work are generated by a NOPA that produces orthogonally polarized but frequency degenerate signal and idler beams for channels $(A, B)$. We emphasize that these beams represent a realization of the entangled state originally discussed by Einstein, Podolsky, and Rosen [42,43]. For the original EPR state, there exist perfect correlations both in position and momentum for two massive particles. In the optical case, the quadrature amplitudes of the electromagnetic field play the roles of position and momentum with a finite degree of correlation for finite NOPA gain, as has been experimentally demonstrated [9] and exploited to realize quantum teleportation [26].

A coherent-state "message" of total amplitude $\epsilon / t$ is encoded in equal measure onto these entangled EPR beams by orienting its polarization at $45^{\circ}$ with respect to the signal and idler polarizations at the mirror $M$ of Fig. 1. To obtain a quantitative statement of the performance of this system, we must include the finite gain of the amplifier as well as various passive losses, which together limit the degree of correlation that can be exploited for communication. Following the analysis of Ref. [9], we find that the $\operatorname{SNR} R_{j}(\Omega)$ for the individual signal and idler photocurrents for propagation and detection in the presence of overall channel efficiency $\xi$ is given by $R_{j}(\Omega)=\xi \epsilon^{2} / 2 G_{q}(\Omega)$, where $G_{q}(\Omega)$ is the detected quantum-noise gain of the amplifier, which can be determined experimentally from measurements of the spectral densities $\Psi_{A, B}(\Omega)$ for the fluctuations of photocurrents for signal and idler beams alone at either detector. Relative to the frequency of the optical carrier determined by the downconversion process in the NOPA, the frequency $\Omega$ specifies the Fourier components of the quadrature-phase amplitudes of signal and idler fields as well as of the coherent field $\epsilon$ [41]. Note that $\xi(0 \leqslant \xi \leqslant 1)$ incorporates the cavity escape efficiency for our NOPA, the propagation efficiency from the NOPA to the detectors, and the homodyne and quantum efficiencies of the balanced detectors themselves [9].

Although the individual fluctuations for channels $(A, B)$ give rise to a level $G_{q}(\Omega)>1$, (that is, greater than the vacuum-state limit of either beam alone), these large fluctuations are correlated in a nonclassical manner and hence can be eliminated by proper choice of the quadrature amplitudes detected at $(A, B)$. As shown in Ref. [9], there is a continuous set of such amplitudes with minimum variance for their difference requiring only that the quadrature-phase angles $\left(\theta_{A}, \theta_{B}\right)$ satisfy $\theta_{A}+\theta_{B}=2 p \pi(p=$ integer $)$. Denoting one such pair by $\left(X_{A}, X_{B}\right)$, we have that

$$
\begin{aligned}
& \left\langle\left[X_{A}(\Omega)-X_{B}(\Omega)\right]\left[X_{A}\left(\Omega^{\prime}\right)-X_{B}\left(\Omega^{\prime}\right)\right]\right\rangle \\
& \quad=V_{-}(\Omega) \delta\left(\Omega+\Omega^{\prime}\right),
\end{aligned}
$$

where $V_{-}(\Omega)$ is a variance that quantifies the degree of correlation between $\left(X_{A}, X_{B}\right)$. Explicit expressions for both $V_{-}(\Omega)$ and $G_{q}(\Omega)$ are given in Ref. [9]. For propagation and detection in the presence of loss, we introduce the quantities $\left(V_{-}^{d}, G_{q}^{d}\right)$ which refer to the variance and quantum noise gain for fictitious fields having propagated with total loss $(1-\xi)$, where the spectral density of the photocurrent fluctuations $\Phi_{-}(\Omega)$ is proportional to $V_{-}^{d}(\Omega)$. Hence, the SNR $R_{d}$ for detection of the message via $i_{-}$is given by $R_{d}$ $=2 \eta \epsilon^{2} / V_{-}^{d}(\Omega)$, where $\eta$ accounts for the propagation and detection efficiency for the message from the mirror $M$ to the photocurrent $i_{A, B}$. Without discussing the general case, here we note simply that for efficient propagation and detection with $(1-\xi) \ll 1$ and for near threshold operation with (analysis frequency $\Omega) \ll\left(\right.$ cavity linewidth $\Gamma$ ), then $G_{q}^{d}(\Omega) \rightarrow 1$ $+\frac{1}{2}(\Gamma / \Omega)^{2} \xi$, while $V_{-}^{d}(\Omega) \rightarrow 2(1-\xi) \ll 1$, so that $R_{d}(\Omega)$ $\rightarrow \eta \epsilon^{2} /(1-\xi)$. Hence in the ideal case with $\eta \rightarrow 1$ and $\xi$ $\rightarrow\left(1-|t|^{2}\right)$, with $t$ as the amplitude transmission coefficient of mirror $M$, we find that the reconstructed message is recovered with the same SNR with which it was originally encoded (namely $R_{d} \rightarrow \epsilon^{2} / t^{2}$ ), while the fluctuations in the individual channels become arbitrarily large $\left(R_{A, B} \sim \Omega^{2} \epsilon^{2} / \Gamma^{2}\right.$ $\rightarrow 0$ for $\Omega / \Gamma \rightarrow 0$ ).

As for the performance as an optical tap, note that the transfer coefficient associated with the detected message at the receiver and with the reflected output field is given by $T_{d}=\left(R_{d}+R_{r}\right) / R_{0}$, where $R_{d}$ is related to $R_{t}$ by way of the propagation and detection efficiency $\eta$ from $M$ to the photocurrents at the receivers. In the present case, we have that

$$
T_{d}=\frac{|r|^{2}}{U_{-}^{r}(\Omega)}+\frac{2 \eta|t|^{2}}{V_{-}^{d}(\Omega)},
$$

with $|r|^{2}$ as the reflectivity of mirror $M\left(|r|^{2}+|t|^{2}=1\right)$ and $U_{-}^{r}(\Omega)$ as the variance of the reflected field. Hence in the ideal case with $\xi \rightarrow\left(1-|t|^{2}\right)$, with $\mathrm{V}_{-}^{d}(\Omega) \rightarrow 2(1-\xi)$ and with $U_{-}^{r}(\eta)=|r|^{2}$, we have that $R_{d} \rightarrow R_{t}$ and $T_{d} \rightarrow 2$. Thus, in addition to providing large quantum fluctuations for secure transmission, the system also acts as a quantum optical tap with a nearly ideal transfer coefficient $T$.

In fact the system can be considered as a realization of the scheme for quantum tapping that was originally suggested by Shapiro [44]. To see this more clearly, recall that the projection of signal and idler fields along the $45^{\circ}$ polarization direction of the message beam results in a squeezed field [41]. Hence, from the perspective of Ref. [44], we are "tapping", the original message field by injecting squeezed light into the normally open (or vacuum) port of mirror $M$. The use of the output of a nondegenerate parametric amplifier allows us subsequently to decompose this squeezed plus coherent field into individually noisy signal and idler fields at polarizer $\mathrm{P}$ for transmission.

\section{EXPERIMENTAL SETUP AND RESULTS}

The general scheme for our experimental implementation of these ideas is shown in Fig. 1, where frequency degenerate but orthogonally polarized signal and idler beams are generated by Type II down conversion in a subthreshold optical parametric oscillator formed by a folded cavity containing an $a$-cut crystal of potassium titanyl phosphate (KTP) that provides noncritical phase matching at $1.08 \mu \mathrm{m}$. The crystal is $10 \mathrm{~mm}$ long, is antireflection coated for both 1.08 and 


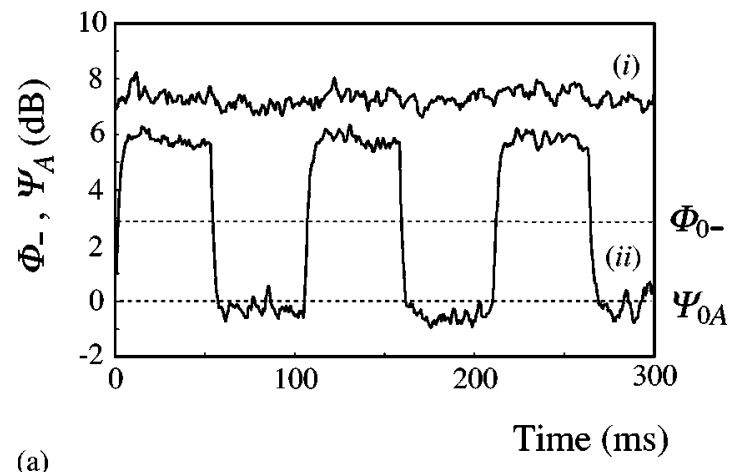

(a)

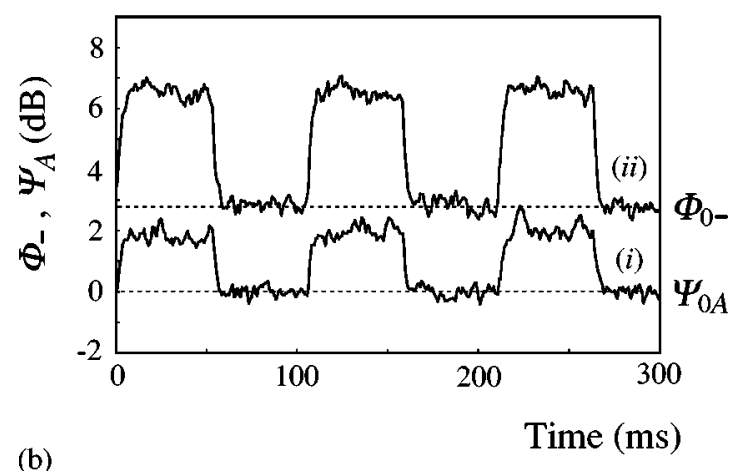

FIG. 2. (a) Signal recovery with correlated quantum states in channels $(A, B)$. Trace i gives the spectral density $\Psi_{A}(\Omega)$ of photocurrent fluctuations for channel $A$ alone as a function of time. Trace ii is the spectral density $\Phi_{-}(\Omega)$ for the combined photocurrent $i_{-}=i_{A}-i_{B}$ from both channels; here the message (coherent beam chopped on and off) clearly emerges. (b) Signal recovery with uncorrelated vacuum fluctuations. Again, trace i gives $\Psi_{A}(\Omega)$ (channel $A$ only) while trace ii gives $\Phi_{-}(\Omega)$ (combined photocurrent $i_{-}$). In (a) and (b) the vacuum-state level $\Psi_{0 A}$ for channel $A$ (signal beam only) and $\Phi_{0-}$ for the combined photocurrent $i_{-}$(dual beam) are shown as dashed lines. Note that $\Psi_{0 A}$ lies $15 \mathrm{~dB}$ above the electronic noise floor. Spectrum analyzer acquisition parameters are as follows: resolution bandwidth $=100 \mathrm{kHz}$, video bandwidth $=100 \mathrm{~Hz}$, analysis frequency $\Omega_{0} / 2 \pi=1.1 \mathrm{MHz}$, and sweep time $=300 \mathrm{~ms}$.

$0.54 \mu \mathrm{m}$, and has a measured harmonic conversion efficiency of $6 \times 10^{-4} / \mathrm{W}$ (single pass) for this geometry. The total intracavity passive losses at $1.08 \mu \mathrm{m}$ are $0.3 \%$ and the transmission coefficient of mirror $M 1$ is $3 \%$. The amplifier is pumped by green light at $0.54 \mu \mathrm{m}$ generated by external frequency doubling of a frequency-stabilized, $\mathrm{TEM}_{00}$-mode Nd:YAP laser [46]. The subthreshold oscillator acts as a narrow-band amplifier (NOPA) which is locked to the original laser frequency with a weak counter-propagating beam. Simultaneous resonance for the orthogonally polarized signal and idler fields is achieved by adjusting the temperature of the KTP crystal around $60^{\circ} \mathrm{C}$ with milliKelvin precision. The pump field at $0.54 \mu \mathrm{m}$ is itself resonant in a separate and independently locked build-up cavity (enhancement $\sim 5 \times)$.

As we have demonstrated in our previous experiments [9], the orthogonally polarized signal and idler fields generated by the NOPA, individually are fields of zero mean values and exhibit large phase insensitive fluctuations. It is in

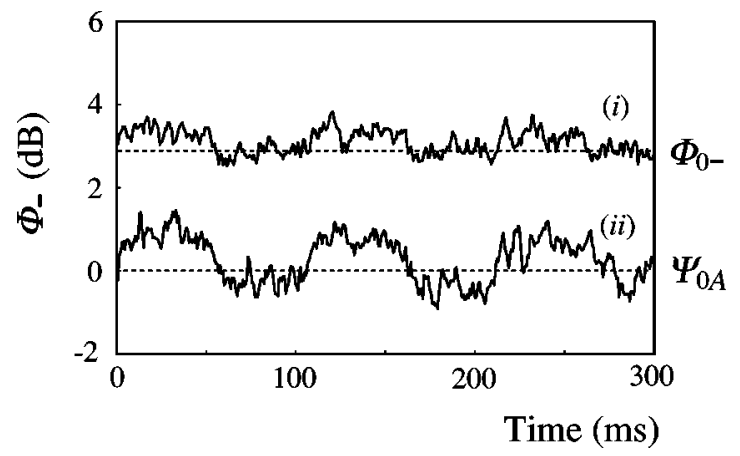

FIG. 3. Spectral density of the photocurrent fluctuations $\Phi_{-}(\Omega)$ for $i_{-}=i_{A}-i_{B}$ for the case when the on-off modulation of the message is encoded with small SNR. Trace $i-$ Uncorrelated vacuum fluctuations. Trace ii-Correlated quantum fluctuations in channels $(A, B)$. The vacuum-state limits $\Psi_{0 A}$ and $\Phi_{0-}$ are indicated. Acquisition parameters are as in Fig. 2.

the midst of this noise that we now hide a message, with this coherent field being combined with the signal and idler fields at the highly reflecting mirror $M$ shown in Fig. $1\left[\left(1-t^{2}\right)\right.$ $\simeq 0.99]$. The coherent beam is injected at $45^{\circ}$ with respect to signal and idler polarizations and is frequency shifted by $\Omega_{0} / 2 \pi=1.1 \mathrm{MHz}$ (single-side band) from the primary laser frequency with the help of a pair of acousto-optic modulators, which are gated "on" and "off" to provide information encoded for transmission. The noisy but correlated signal and idler beams together with the coherent information are then separated by a polarizer $P$, transmitted independently over the two channels $(A, B)$, and then directed to two separate balanced homodyne detectors for measurements of their individual quadrature-phase amplitudes and their mutual correlations. The local oscillators for the two balanced homodyne detectors originate from the laser at $1.08 \mu \mathrm{m}$; their phases can be independently controlled by mirrors mounted on piezoelectric transducers. The spectral densities of the photocurrents for the two channels $(A=$ signal, $B$ $=$ idler) are defined by

$$
\Psi_{A, B}(\Omega)=\int\left\langle i_{A, B}(t) i_{A, B}(t+\tau)\right\rangle e^{i \Omega \tau} d \tau
$$

and are recorded by a rf spectral analyzer, as is the spectral density

$$
\Phi_{-}(\Omega)=\int\left\langle i_{-}(t) i_{-}(t+\tau)\right\rangle e^{i \Omega \tau} d \tau
$$

for the difference photocurrent $i_{-} \equiv i_{A}-i_{B}$.

In Figs. 2 and 3 we present results from a series of measurements of these various spectral densities. First of all, in Fig. 2(a), trace i gives the spectral density $\Psi_{A}$ for channel $A$ alone with an injected message and with the amplifier turned on to generate large $(\sim 7 \mathrm{~dB})$ phase insensitive noise above the vacuum-state level $\Psi_{0 A}$ (indicated by a dashed line in Fig. 2) for the signal beam. A similar trace is obtained for the spectral density $\Psi_{B}$. By contrast, trace ii in Fig. 2(a) gives the spectral density $\Phi_{-}$for the difference photocurrent $i_{-}$, with the phases of the local oscillators adjusted for minimum 
noise and maximum coherent signal. In this trace, the coherent message that was completely obscured in trace i emerges with high signal-to-noise ratio. Note that in trace ii the correlated quantum fluctuations for signal and idler fields are subtracted to approximately $0.4 \mathrm{~dB}$ below the vacuum-noise level $\Psi_{0 A}$ of the signal beam alone (and likewise for the idler), indicating an improvement in SNR over a conventional single-channel communication scheme with a classical light source.

To complete the discussion, we present in Fig. 2(b) results obtained with the amplifier turned off (that is, uncorrelated vacuum-state inputs for signal and idler fields, that are combined with the coherent message information at mirror $M$ ). Trace $\mathrm{i}$ shows the result for the signal beam alone $\left(\Psi_{A}\right)$, where again the noise floor $\Psi_{0 A}$ is from the vacuum fluctuations of the signal beam; a similar trace is obtained for the idler beam $\left(\Psi_{B}\right)$. Trace ii gives the corresponding result for $\Phi_{-}$for the combined signal and idler photocurrents when the amplifier is off. Note that this trace represents the best possible SNR with which the encoded information can be recovered when correlated classical noise sources are employed since here the (uncorrelated) vacuum fluctuations of signal and idler beams set an ultimate noise floor $3 \mathrm{~dB}$ above $\Psi_{0 A}$ (that is, $\Phi_{0-}=2 \Psi_{0 A}$ ) [47]. On comparing traces ii in Figs. 2(a) and 2(b), we see that the correlated quantum fluctuations of signal and idler fields brought about by parametric amplification result in an improvement in SNR of $3.2 \mathrm{~dB}$ relative to that possible with classical noise sources.

The improvement in SNR with correlated quantum fields over classical fields in our two-channel communication scheme can be of utility especially when the message is so weak that the SNR is poor for transmission with correlated classical sources (that is, for the case where vacuum noise dominates the encoded message). This situation is illustrated in Fig. 3, where we plot $\Phi_{-}$for the two cases without [(trace i) and with (trace ii)] correlated quantum fields [47]. Relative to Fig. 2, here the coherent beam has been attenuated resulting in a smaller SNR for the message. Indeed in trace $\mathrm{i}$, this information is "buried" by the vacuum noise $\Phi_{0-}$ associated with independent vacuum fluctuations in channels $A$ and $B$; recovery of the encoded information is poor. On the other hand, as shown in trace ii, when correlated quantum fields are employed, there is a reduction in the noise floor by more than $3 \mathrm{~dB}$, which makes possible improved recovery of the encoded information, with the recovery here limited by losses in propagation and detection [9].

As for the actual performance with respect to optical tapping, our system falls far short of the projected possibilities discussed in the preceding section because of an unfortunate mismatch between the transmissivity $|t|^{2}$ for mirror $M$ and the overall system efficiency $\xi$. In quantitative terms, recall that the transfer coefficient $T$ for encoding information from the input beam to the reflected and transmitted beams at $M$ is given by $T \equiv\left(R_{r}+R_{t}\right) / R_{0}$ whereas the transfer coefficient for the detected message photocurrent and the reflected signal field is $T_{d} \equiv\left(R_{r}+R_{d}\right) / R_{0}$ as given explicitly in Eq. (2). For the propagation and detection efficiencies in our experiment ( $\xi \simeq 0.65$ and $\eta \simeq 0.75$ ), these transfer coefficients are optimized for mirror transmission $|t|^{2} \sim 0.5$ for $M$. In our arrangement we have instead $|t|^{2}=0.01$, with the inferred result that $T_{d} \simeq 1.02$, which is only marginally in the quantum domain.

In the experiment described here, the receiver uses a local oscillator (LO) that originates from the fundamental frequency of the same laser that generated the pump beam for the NOPA. This LO is necessary for proper detection of the quadrature amplitudes of the nonclassical beams and of the message, since it provides a phase reference that follows phase fluctuations of the NOPA's pump beam. In practice, as the stability of the available lasers improve, one should consider schemes for which the measurement is carried out with nominally independent lasers for the LO and for the source. For example, one might employ a stabilized laser diode as a reference to phase-lock lasers both at the sender and at the receiver, where the laser diode could be widely distributed through optical fibers. Alternatively, Ralph has analyzed a scheme in which the local oscillators are transmitted and recovered as part of the overall protocol [35].

\section{COMPARISON WITH OTHER DUAL-BEAM SCHEMES}

It is perhaps obvious that the degree of immunity to interception for a two channel scheme such as we have discussed, is related to the degree of excess fluctuations for each individual beam. For the demonstration in Ref. [48], the excess noise that is used to "hide" the encoded information in each beam comes from some artificial unrelated source. Unfortunately such uncorrelated excess fluctuations also add noise to the coincidence signal in the recovery of the message, even though the added noise scales differently as a function of photon number for single-beam measurements (linearly) and for dual-beam measurements (quadratically). Hence larger background noise, which better "hides" the encoded information also brings larger added noise in the extraction of the message. Because of the quadratic dependence on the total photon number for the extra noise added in coincidence detection, this scheme is best suited to low-light level transmission, as demonstrated in the pioneering experiment by Hong et al. [48].

The situation is quite different for the quadrature-phase amplitudes of the correlated signal and idler fields generated by the NOPA. As the NOPA is pumped harder and the threshold for parametric oscillation is approached, the gain of the amplifier increases, as do the excess fluctuations of the signal and idler fields. However, the correlation between the fluctuations of the signal and idler beams also improves, giving rise to even better SNR for the recovered signal. The key point is that the large fluctuations in the signal and idler beams needed for immunity to interception are intrinsic and do not add extra noise to the recovered signal but, on the contrary, serve to reduce the noise in $i_{-}$as the gain of the amplifier increases. In the end, the SNR for the recovered message is arbitrated by the imperfect correlation resulting from finite gain and from passive losses in propagation and detection. On the other hand, this dependence provides a powerful means to detect eavesdropping because unauthorized extraction of signal or idler fields from channels $A$ or $B$ results in a reduction of the detected correlation and hence an 
increase in the noise floor of the recovered message. Note that unauthorized extraction of information from both channels by way of a quantum optical tap [44] or a quantum nondemolition measurement [11] can likewise be detected because of the unavoidable increase of fluctuations for the orthogonal quadrature-phase amplitudes $\left(\theta_{A, B}+\pi / 2\right)$ of the two channels. Furthermore, these quantum eavesdropping schemes can be defeated in large measure by random switching of the phases of the message, signal, and idler beams as discussed below.

Our system also offers advantages with respect to the (classical) digital Vernan cipher, where a message is decomposed in two correlated random signals and transmitted over two one-way channels. Although this system seems to be similar to ours in the sense that is also secure provided the eavesdropper has access to one channel only, the situation is different if the eavesdropper can split a small fraction of both channels since in the classical case, this can be done without the knowledge of the receiver. However, in our system the eavesdropper cannot choose arbitrarily the reflectivity of any "beamsplitter" used for extraction from the two channels since in the quantum case, the fraction of the beams extracted should be big enough so that the signal-to-noise ratio for the intercepted message is greater than one. But if this is the case, then unavoidable extra "noise" added to the transmitted beams by the open port of the "beamsplitter" degrades the signal-to-noise ratio of the message at the legitimate receiver, thus revealing the unauthorized intervention during transmission.

One might attempt to circumvent this difficulty by employing a quantum extraction procedure, such as quantum nondemolition detection [11] of the quadrature amplitudes in channels $(A, B)$. Although the signal-to-noise ratio $R_{d}$ at the receiver would not in this case be degraded by an ideal eavesdropper, the unauthorized intervention could nonetheless be discovered because of the injection of large fluctuations ("backaction" noise) in the quadrature orthogonal to that in which signal information is stored, as previously noted.

\section{EXTENSIONS VIA RANDOM-PHASE SWITCHING}

One way an eavesdropper Eve could access the signal and idler beams without the knowledge of the legitimate receiver is if she can intercept both channels completely, detect in the same manner as does the legitimate receiver (i.e., Eve should also have access to a local oscillator phase stable with respect to that of sender and receiver) and retransmit the beams in the same way as the legitimate sender. Because of this possibility, our protocol as described is certainly not secure, in contrast to the protocols for discrete variables [37-39]. However, we suggest that simple extensions of our protocol might lead to significant enhancements in security.

If the goal were to achieve quantum key distribution, one idea is to make straightforward adaptations of the protocols introduced by Bennett and colleagues for the discrete case, as in Refs. [34-36]. Here, we propose that the sending station (Alice) and receiving station (Bob) make random choices for the set of phases of the coherent message beam, as well as for the signal and idler beams. Recall that the variance $V_{-}(\Omega)$ of Eq. (1) is the minimum possible and applies only for the choice of quadrature-phase angles $\left(\theta_{A}, \theta_{B}\right)$ for the signal and idler beams that satisfy $\theta_{A}+\theta_{B}=2 p \pi$ ( $p$ =integer). For definiteness, assume the following two choices.

(1) $\left(\theta_{A}^{0}, \theta_{B}^{0}\right)$, with $\theta_{A}^{0}+\theta_{B}^{0}=0$ and corresponding quadrature amplitudes $\left(X_{A}, X_{B}\right)$.

(2) $\left(\theta_{A}^{\pi / 2}=\theta_{A}^{0}+\pi / 2, \theta_{B}^{\pi / 2}=\theta_{B}^{0}+\pi / 2\right)$ and corresponding quadrature amplitudes $\left(Y_{A}, Y_{B}\right)$.

In the first case, the minimum variance $V_{-}(\Omega)$ results for the combination $\left(X_{A}-X_{B}\right)$, while in the second case, the combination $\left(Y_{A}+Y_{B}\right)$ has minimum variance. This is because $Y_{B} \rightarrow-Y_{B}$ is equivalent to the shift $\theta_{B}^{\pi / 2} \rightarrow \theta_{B}^{\pi / 2}+\pi$, so that $\theta_{A}^{\pi / 2}+\theta_{B}^{\pi / 2}+\pi=2 \pi$.

With these definitions, Alice at the sending station (randomly) makes one of two choices.

(1) Phase 0: Set the quadrature-phase angles $\left(\theta_{A}, \theta_{B}\right)$ to $\left(\theta_{A}^{0}, \theta_{B}^{0}\right)$ and the phases $\beta_{A, B}=\beta_{A, B}^{0}$ for the coherent message beam $|\alpha\rangle=|\alpha| \exp [i \beta]$ corresponding to the $X$ quadratures of $(A, B)$.

(2) Phase $\pi / 2$ : Set $\left(\theta_{A}, \theta_{B}\right)$ to $\left(\theta_{A}^{\pi / 2}, \theta_{B}^{\pi / 2}\right)$ and $\beta_{A, B}$ $=\beta_{A, B}^{\pi / 2}=\beta_{A, B}^{0} \pm \pi / 2$ corresponding to the $Y$ quadratures.

The encoded message (which could consist of $|\alpha|$ $=\left[a_{0}, a_{1}\right]$ for a binary transmission) is sent to Bob's receiving station precisely as in Fig. 1. Bob must then choose the appropriate phases $\left(\phi_{A}, \phi_{B}\right)$ for his local oscillators $\left(\mathrm{LO}_{A}, \mathrm{LO}_{B}\right)$ to detect quadrature amplitudes such that the spectral density $\Phi_{-}(\Omega)$ for the difference photocurrent $i_{-}$ $\equiv i_{A}-i_{B}$ is minimized and the signal maximized. In the case Phase 0 , denote the local oscillator settings as $\left(\phi_{A}^{0}, \phi_{B}^{0}\right)$, in correspondence to the detection of $\left(X_{A}, X_{B}\right)$ with minimum variance $V_{-}(\Omega)$. On the other hand, for the case Phase $\pi / 2$, the local oscillator phases $\left(\phi_{A}, \phi_{B}\right) \rightarrow\left(\phi_{A}^{\pi / 2}, \phi_{B}^{\pi / 2}\right)=\left(\phi_{A}^{0}\right.$ $\left.+\pi / 2, \phi_{B}^{0}+3 \pi / 2\right)$, in correspondence to the detection of $\left(Y_{A},-Y_{B}\right)$ with minimum variance. In both cases, the encoded message would be recovered with maximum signalto-noise ratio. Note that precisely such a switching protocol was implemented in our prior experiment of Ref. [9] with results as stated for the variances.

Of course, $B o b$ does not know in advance which choice $[0, \pi / 2]$ Alice will have made for any given transmission. Hence, he makes a random selection between the alternatives $\left(\phi_{A}^{0}, \phi_{B}^{0}\right)$ and $\left(\phi_{A}^{\pi / 2}, \phi_{B}^{\pi / 2}\right)$, recovering the message in some cases but not others. After a series of transmissions, Alice and $B o b$ communicate publicly about their choice of bases, keeping measurement results only when their choices coincide.

Now, if an eavesdropper Eve attempts to intervene (either by a strategy of partial tapping or by one of complete interception and rebroadcast), she will necessarily increase the noise level and error rate at $B o b$ 's receiving station. The random switching of the phases $\left(\theta_{A}, \theta_{B}\right)$ by Alice forces Eve to make a guess as to the correct quadratures $\left(\delta_{A}, \delta_{B}\right)$ to be detected. Having made a choice, information about the orthogonal quadrature is lost. Of course, rather than homodyne detection, she could choose to employ heterodyne detection to gain information about the full complex amplitude. How- 
ever, relative to homodyne detection, heterodyne detection brings a well-known penalty of a $3 \mathrm{~dB}$ reduction in signalto-noise ratio [49].

While it is beyond the scope of the current paper to make any claims about the quantitative limits to the information that Eve might access or about the absolute ability of Alice and $B o b$ to detect her presence, we do suggest that these would be interesting questions to investigate. There are certainly intervention strategies beyond those that we have mentioned that a cunning Eve would want to consider, such as an adaptive strategy for adjusting the phases $\left(\delta_{A}, \delta_{B}\right)$ during the duration of the transmission of any given message [50]. Likewise, in any real-world setting, overcoming the deleterious effects of losses in propagation from Alice to $B o b$ will be an overriding consideration. The question of preserving the entanglement of the initial EPR state in the face of such losses is a fascinating one for continuous quantum variables.
Although initial attempts have been made to develop error correcting quantum codes for continuous variables [14-16], no adequate solution seems to yet have been found. Finally, it would be of interest to analyze the case where only one of the two correlated beams is sent to Bob, with Alice retaining the other.

\section{ACKNOWLEDGMENTS}

The authors gratefully acknowledge the comments of J.H. Shapiro, who pointed out the connection between our experiment and that of Ref. [44], and we thank S. L. Braunstein and $\mathrm{H}$. Mabuchi for critical discussions. This work was supported by the Office of Naval Research, by the National Science Foundation, and by DARPA via the QUIC administered by the Army Research Office.
[1] H. Takahasi, Adv. Commun. Syst. 1, 227 (1965).

[2] H. P. Yuen and J. H. Shapiro, IEEE Trans. Inf. Theory IT-24, 657 (1978).

[3] C. M. Caves and P. D. Drummond, Rev. Mod. Phys. 66, 481 (1994); H. P. Yuen and M. Ozawa, Phys. Rev. Lett. 70, 363 (1993).

[4] C. M. Caves, Phys. Rev. D 23, 1963 (1981); 26, 1817 (1982); C. M. Caves et al., Rev. Mod. Phys. 52, 341 (1980).

[5] M. Xiao, L. A. Wu, and H. J. Kimble, Phys. Rev. Lett. 59, 278 (1987); P. Grangier, R. E. Slusher, B. Yurke, and LaPorta, ibid. 59, 2153 (1987).

[6] M. Xiao, L. A. Wu, and H. J. Kimble, Opt. Lett. 13, 476 (1988).

[7] E. S. Polzik, J. Carri, and H. J. Kimble, Phys. Rev. Lett. 68, 3020 (1992).

[8] Z. Y. Ou, S. F. Pereira, and H. J. Kimble, Phys. Rev. Lett. 70, 3239 (1993).

[9] Z. Y. Ou, S. F. Pereira, H. J. Kimble, and K. C. Peng, Phys. Rev. Lett. 68, 3663 (1992); Z. Y. Ou, S. F. Pereira, and H. J. Kimble, Appl. Phys. B: Photophys. Laser Chem. 55, 265 (1992).

[10] J. F. Roch, G. Roger, P. Grangier, J. M. Courty, and S. Reynauld, Appl. Phys. B: Photophys. Laser Chem. 55, 291 (1992).

[11] For a review, see P. Grangier, J. A. Levenson, and J.-P. Poizat, Nature (London) 396, 537 (1998).

[12] J. Ph. Poizat and P. Grangier, Phys. Rev. Lett. 70, 271 (1993).

[13] S. Lloyd and S. L. Braunstein, e-print quant-ph/9810082.

[14] S. Lloyd and J. J. E. Slotine, Phys. Rev. Lett. 80, 4088 (1998).

[15] S. L. Braunstein, Phys. Rev. Lett. 80, 4084 (1998).

[16] S. L. Braunstein, Nature (London) 394, 47 (1998).

[17] S. Parker, S. Bose, and M. B. Plenio, e-print quant-ph/9906098.

[18] L.-M. Duan, G. Giedke, J. I. Cirac, and P. Zoller, e-print quant-ph/9912017.

[19] L. Vaidman, Phys. Rev. A 49, 1473 (1994).

[20] S. L. Braunstein and H. J. Kimble, Phys. Rev. Lett. 80, 869 (1998).

[21] T. C. Ralph and P. K. Lam, Phys. Rev. Lett. 81, 5668 (1998).
[22] T. Opatrny, G. Kurizki, and D.-G. Welsch, e-print quant-ph/9907048.

[23] P. van Loock, S. L. Braunstein, and H. J. Kimble, Phys. Rev. A (to be published).

[24] A. S. Parkins and H. J. Kimble, J. Opt. Soc. Am. B 1, 496 (1999); available as e-print quant-ph/9904062; and submitted, 1999, e-print quant-ph/9909021.

[25] S. L. Braunstein and H. J. Kimble, Phys. Rev. A 61, 042302 (2000).

[26] A. Furusawa, J. Sorensen, S. L. Braunstein, C. Fuchs, H. J. Kimble, and E. S. Polzik, Science 282, 706 (1998).

[27] S. L. Braunstein, C. A. Fuchs, and H. J. Kimble, J. Mod. Opt. 47, 267 (2000).

[28] United States Patent No. 5339 182, Method and Apparatus for Quantum Communication Employing Nonclassical Correlations of Quadrature-Phase Amplitudes, issued 1994, H. J. Kimble, Z. Y. Ou, and S. F. Pereira.

[29] C. H. Bennett, G. Brassard, and A. K. Ekert, Sci. Am. 267, 50 (1992).

[30] J. D. Franson and B. C. Jacobs, Electron. Lett. 31, 232 (1995).

[31] G. Ribordy, J. D. Gautier, N. Gisin, O. Guinnard, and H. Zbinden, J. Mod. Opt. 47, 517 (2000).

[32] R. J. Hughes, G. L. Morgan, and C. G. Peterson, J. Mod. Opt. 47, 533 (2000).

[33] P. D. Townsend, Opt. Fiber Technol.: Mater., Devices Syst. 4, 345 (1998).

[34] M. Hillery, e-print quant-ph/9909006.

[35] T. C. Ralph, e-print quant-ph/9907073.

[36] M. D. Reid, e-print quant-ph/9909030.

[37] For a recent review of security proofs in quantum cryptography, see C. H. Bennett and P. W. Shor, Science 284, 747 (1999).

[38] H.-K. Lo and H. F. Chau, Science 283, 2050 (1999).

[39] D. Mayers, e-print quant-ph/9802025.

[40] S. M. Barnett and P. Knight, J. Opt. Soc. Am. B 2, 467 (1985).

[41] H. J. Kimble, in Fundamental Systems in Quantum Optics, edited by J. Dalibard, J. M. Raimond, and J. Zinn Justin (Elsevier, Amsterdam, 1992), p. 545ff. 
[42] A. Einstein, B. Podolsky, and N. Rosen, Phys. Rev. 47, 777 (1935).

[43] M. D. Reid and P. D. Drummond, Phys. Rev. Lett. 60, 2731 (1988); M. D. Reid, Phys. Rev. A 40, 913 (1989).

[44] J. H. Shapiro, Opt. Lett. 5, 351 (1980).

[45] C. H. Bennett and S. J. Wiesner, Phys. Rev. Lett. 69, 2881 (1992).

[46] Z. Y. Ou, S. F. Pereira, E. S. Polzik, and H. J. Kimble, Opt. Lett. 17, 640 (1992).
[47] In Figs. 2 and 3, $\Phi_{-}=1.9 \Psi_{0, A}(2.8 \mathrm{~dB}$ above $)$ due to a slight imbalance $(0.1 \mathrm{~dB})$ between the $(A, B)$ detectors and to a small contribution $(0.1 \mathrm{~dB})$ from detector thermal noise.

[48] L. Mandel, J. Opt. Soc. Am. B 1, 108 (1984); C. K. Hong, S. R. Friberg, and L. Mandel, Appl. Opt. 24, 3877 (1985).

[49] E. Arthurs and J. L. Kelly, Jr., Bell Syst. Tech. J. 44, 725 (1965).

[50] H. M. Wiseman, Phys. Rev. Lett. 75, 4587 (1995); H. M. Wiseman and R. B. Killip, Phys. Rev. A 57, 2169 (1998). 